\title{
Gradhiva
}

GRADHIV

Revue d'anthropologie et d'histoire des arts

$14 \mid 2011$

Carl Einstein et les primitivismes

\section{Art exotique, 1930}

Exposition à la galerie du Théâtre Pigalle à Paris

\section{Carl Einstein}

Traducteur : Isabelle Kalinowski

\section{OpenEdition}

\section{Journals}

Édition électronique

URL : http://journals.openedition.org/gradhiva/2204

DOI : $10.4000 /$ gradhiva.2204

ISSN : $1760-849 x$

\section{Éditeur}

Musée du quai Branly Jacques Chirac

\section{Édition imprimée}

Date de publication : 30 novembre 2011

Pagination : 194-197

ISBN : 978-2-35744-046-3

ISSN : 0764-8928

Référence électronique

Carl Einstein, « Art exotique, 1930 », Gradhiva [En ligne], 14 | 2011, mis en ligne le 30 mai 2012, consulté le 19 avril 2019. URL : http://journals.openedition.org/gradhiva/2204 ; DOI : 10.4000/ gradhiva.2204 


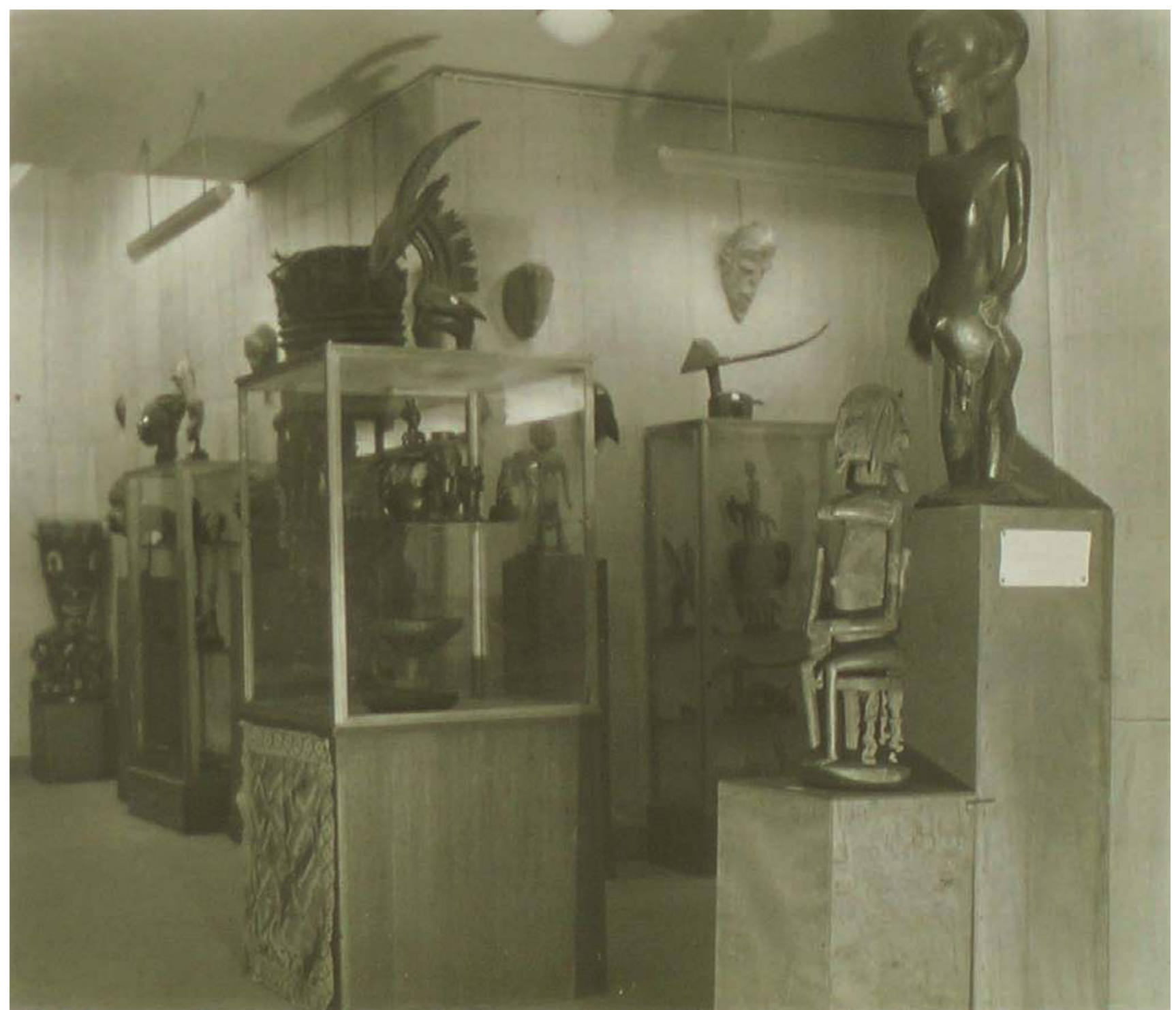

Fig. 1 Vue de l'exposition d'art africain et d'art océanien à la galerie du Théâtre Pigalle en 1930 @ collection Société française de photographie. DR. 


\section{Art exotique. Exposition à la galerie du Théâtre Pigalle à Paris}

Ce texte, traduit ici pour la première fois en français, parut initialement en allemand dans le journal Die Kunstauktion, le 2 mars 1930.

La galerie du Théâtre Pigalle présente une exposition d'art africain et océanien. Il serait souhaitable que celle-ci incite à ne plus aborder le domaine gigantesque de cet art d'un point de vue purement esthétique, mais surtout archéologique. Il est temps d'opérer des distinctions dans cette sphère immense, du point de vue des styles et de l'histoire de l'art, et de réaliser des monographies particulières. Mais pour cela, certaines conditions doivent être réunies. Il faut d'abord une mythologie africaine comparée et une histoire des religions de l'Afrique. Il faudrait également établir les traditions et les mythes itinérants des grands empires. La division en aires culturelles à laquelle on a volontiers recours devrait alors s'avérer insuffisante car, en Afrique, des strates très différentes se superposent. Une méthode tout aussi contestable est celle qui consiste à présumer, dans les datations, que la forme la plus simple est la plus ancienne. Les naturalismes magiques sont souvent plus anciens que le style qui leur a succédé. Il est certain que, dans les régions provinciales ou relativement protégées des migrations, des formes plus anciennes ont été préservées, sur un mode conservateur. Il faudrait ensuite parvenir à une cristallisation des formes de religion les plus anciennes à partir des multiples cultes magiques dans lesquels ces religions africaines ont dégénéré.

Il est clair que l'art africain n'a pas manqué d'éveiller la participation des artistes modernes. Il n'est guère d'art qui ait clarifié si nettement le problème du volume plastique. D'un autre côté, on voit bien avec l'art moderne, Picasso en particulier, combien la figuration magique est proche de nous. On recommence à inventer des figures qui ne sont pas placées sous la dépendance des naturalismes.
Cen'est pas icile lieu d'expliquer la signification religieuse de ces statues, que nous considérons comme le siège des esprits. La statue attire en son sein les esprits des morts; d'un autre côté, elle perd sa valeur magique avec le passage du mort du côté du dieu éloigné des hommes. C'est là une des raisons pour lesquelles tant de statues ont été jetées ou abandonnées.

Cette statuaire africaine est de prime abord statique. On pense à une parenté avec la sculpture égyptienne. L'âme ombre, l'itongo de l'Africain, rappelle le ka des Égyptiens. L'Africain dispose de quelques motifs fondamentaux extraordinairement importants qui sont aussi incroyablement heureux d'un point de vue plastique. C'est le pilier phallique, qui est aussi un signe du soleil masculin lié à la forme du crâne féminin (hutte des esprits). La caractéristique de ce motif est d'être confiné à la zone sexuelle; cela peut se comprendre, car l'abandon de celle-ci implique une réduction de la force vitale de la tribu. Le pilier phallique possède la même signification que les monolithes que nous trouvons aussi en Afrique. Le motif du crâne se retrouve dans les têtes juju du Bénin et du Cameroun, les crânes-cuves des Pahouins, les coupes-crânes des Ba-Kuba. Le pilier et le crâne présentent en même temps un important contraste plastique. Un second motif fondamental : le squelette. Les hommes exotiques désignent souvent la sculpture et le cadavre sous un même terme. Des exemples sont fournis en Océanie par les statuettes de bois de l'île de Pâques ou par les figures d'ancêtres de Nouvelle-Guinée. L'art africain est entièrement dominé par le motif antique de la mort. La mort est en un sens l'idée fixe de l'Africain, qui regarde le vivant tout entier du point de vue de la mort. Les vivants vivent avec les morts et les statues sont des doubles des morts, grâce 
auxquelles on s'assure un pouvoir sur les esprits, les itongo. Ces statues sont perçues sur un mode entièrement animiste, mais comme elles sont inventées à partir de la mort, leur attitude est résolument statique. Cet art est plutôt non naturaliste, car sa fonction était de rendre visible l'insaisissable, l'invisible, à savoir les morts. On peut distinguer : les figures d'ancêtres, les statues magiques, puis les produits de l'art de cour et de l'art profane. Dans le Kassaï, on distingue les mikisi mihake, c'est-à-dire des statuettes dotées de substances magiques, et les mikisi mikasi, les statuettes qui rappel- lent la mémoire des morts; on les appelle aussi bimweholo, l'apparence.

Le grand drame religieux du totémisme est, logiquement, la métamorphose; car un esprit peut établir sa demeure dans différents objets. C'est là qu'on a créé ces extraordinaires vêtements pourvus de masques qui sont à leur tour des démons. Le masque lui-même, en particulier le masque qui peut être relevé au-dessus de la tête, reprend lui aussi le vieux motif de la demeure des esprits. Mentionnons encore rapidement les statues hermaphrodites que l'on trouve surtout au Soudan, ou

A propos de l'Exposition de la Galerie Pigalle
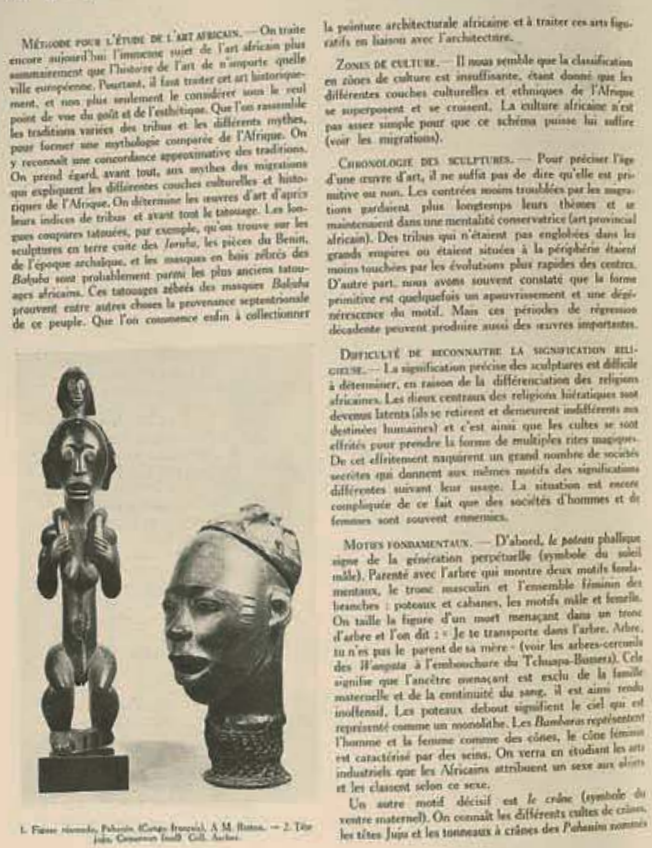

104

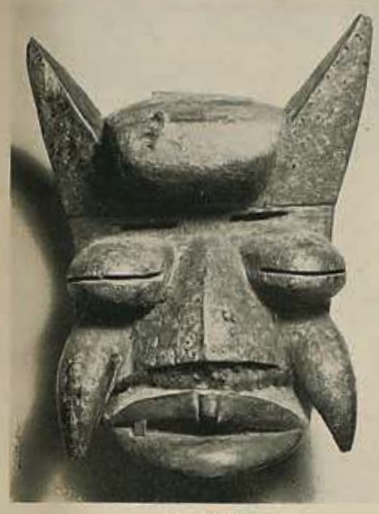

Muspen Dam. Calli de Mirrt

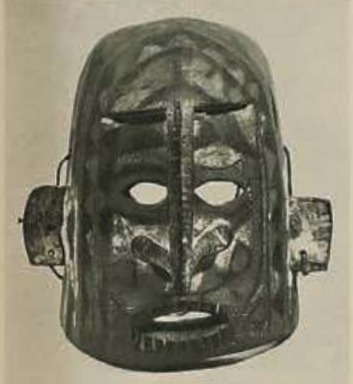

Marere Uramal. A M. Ration

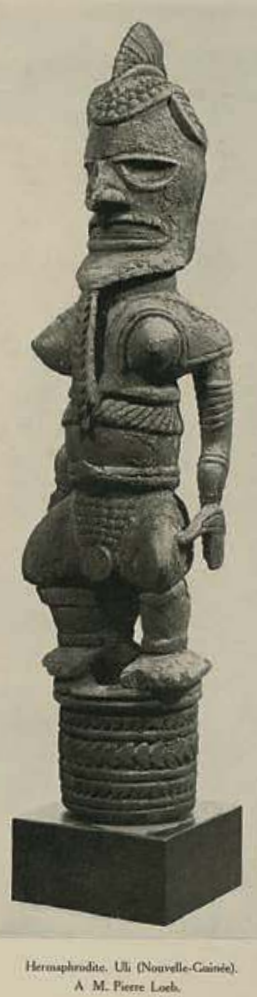

Fig. 2 Article de Carl Einstein, «À propos de l'exposition de la galerie Pigalle », Documents n² 2, vol. II, 1930. Photo Alberto Ricci. 
les statues bisexuelles du Nouveau-Mecklembourg, dites uli. L'état d'hermaphrodite était apparemment tenu pour une forme d'accomplissement divin, dont la perte était en quelque sorte la conséquence d'un péché. Les figures hermaphrodites incarnentle degré suprême de vitalité et l'actus generationis lui-même. Soulignons que cet art est resté associé à la zone sexuelle. Ainsi, les outils aussi étaient masculins ou féminins.

Cette exposition montre de façon intéressante et convaincante les différentes strates stylistiques africaines. La thèse d'un isolement de l'Afrique est une ineptie dépassée. On peut bien plutôt observer un éclectisme africain (voir l'art côtier de l'Ouest ou les masques du plateau Makondé ou du Mozambique). Pourtant, la force de la culture africaine se traduit dans le fait qu'elle a puissamment africanisé toutes les influences et les motifs étrangers. Les liens entre la peinture de l'Ouest de l'Espagne et la peinture africaine sont peu à peu avérés, et ces relations semblent s'étendre jusqu'à l'Afrique du Sud. Au demeurant, il faut se garder de les situer à une époque trop ancienne; car il faut toujours compter avec le conservatisme africain.

Il a sans doute existé ensuite une strate d'art africain qui correspond à peu près à la couche préhellénique. L'art ancien de l'Est du Soudan entretenait certainement des liens étroits avec l'art hittite et l'art de l'Arabie du Sud. Nous pensons que l'influence de l'Égypte s'exerça durablement; c'est au demeurant par son intermédiaire que furent transmis des motifs méditerranéens qui avaient déjà été retravaillés en Afrique du Nord. À l'est, on trouve des masques plats qui ressemblent aux figurines plates des Cyclades. Sur les calebasses de la Côte d'Or sont dessinées les mêmes images frontales que sur les reliefs babyloniens ou les miroirs étrusques. La fonte de métal à la cire perdue est apparue au même moment en Élam et dans l'Égypte ancienne. L'influence de l'Arabie du Sud préislamique et des Phéniciens s'est étendue jusqu'en Afrique du Sud; en témoignent les bâtiments et les sculptures du Zimbabwe, qui sont malgré tout de conception africaine. L'art de l'Est du Soudan fut ensuite pratiquement mis à l'écart par des éleveurs hamites. Les Soudanais de l'Est qui migrèrent vers l'ouest apportèrent avec eux des formes et un monde de motifs relativement unifiés. Nous pensons retrouver leur héritage formel, à l'ouest, principalement chez les Pahouins, porteurs d'un classicisme, et chez les tribus de l'Ogooué. Nous sommes également d'avis que, dans l'histoire de l'art, on a trop isolé les œuvres du Bénin. Le style de cet art s'étend de la Sierra Leone à l'embouchure du Congo, le Majombe. Nous rencontrons ensuite une strate qui correspond au Moyen Âge africain, dans laquelle se font surtout sentir les influences copte et byzantine. Plus tard est arrivée une vague musulmane qui fut fatale pour l'art africain et détruisit des biens culturels insignes ou, plus exactement, leur fit perdre leur spécificité. Les formes islamiques surjouent l'ornementation africaine et, cette fois, les Nègres ne furent pas vraiment en mesure de surmonter ces influences extérieures. L'héritage africain authentique fut conservé dans la province, dans les régions reculées et protégées du Cameroun et dans les forêts du Kassaï et Sankuru. C'est là que ressuscita pour la dernière fois un grand archaïsme magique. Les vieux motifs fondamentaux de la sculpture africaine s'étaient à nouveau imposés; mais les royaumes s'écroulent et les religions se divisent en cultes magiques, la divinité centrale est oubliée et l'idée fixe de la mort et des morts dévaste l'Afrique. Elle devient stérile.

Cette exposition de Paris montre quelque chose que les Français ne connaissaient pas auparavant, la grande Renaissance camerounaise, et ce sera peut-être une découverte pour la France, qui aimait jusque-là avant tout les fines pièces classiques de la côte ivoirienne et des Pangwe.

L'art africain meurt avec la religion africaine et, au demeurant, celle-ci contient déjà les germes d'une décadence. L'esprit du mort n'a peut-être pas toujours besoin de la statue. Il peut établir sa demeure dans n'importe quelle plante ou n'importe où ailleurs. Ce n'est pas tout : l'Africain aime oublier les ancêtres qui l'assaillent. Il les fuit dans l'exogamie. Le culte de la statue de l'ancêtre atteint peut-être son point culminant avec le petit-fils, car il est lui-même le grand-père ressuscité. Peu à peu, cependant, la force magique de ces sculptures s'épuise et elles sont oubliées. Ces raisons ont aussi contribué à l'immense décadence de ce grand art que fut l'art africain. 